\title{
The Registration and Presentation of MR and PET Fusion Imaging
}

\author{
${ }^{1}$ San-Chau Hwang, ${ }^{1}$ Jyh-Horng Chen, ${ }^{23}$ Jason J.S. Lee, and ${ }^{3}$ Shin-HwaYeh1. \\ 1. Department of Electrical Engineering, National Taiwan University \\ 2. Division of Radiological Sciences National Yang-Ming University \\ 3. National Positron Emission Tomography and Cyclotron Center \\ Taipei, Taiwan
}

\begin{abstract}
We have developed a method that allow us to register 3D multi-modalities imaging sets automatically. This method use the N-bar landmarks(Fig. 1) to register MR and PET imaging. A WINDOWS-95 based software for the presentation of MR and PET fusion imaging has been designed. With friendly operation, this software can interactively display the registered imaging at an arbitrary slice in real time.
\end{abstract}

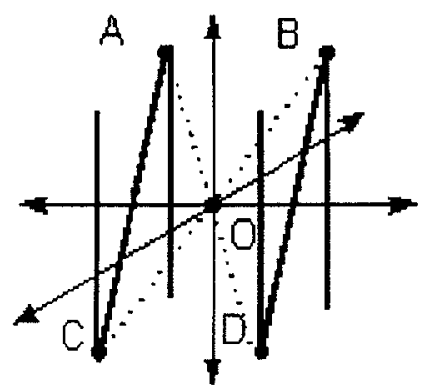

Fig. 1 The N-bar landmarks

\section{Introduction}

Clinical diagnosis is often supported by several imaging modalities. For examples, the anatomic and functional information can be individually obtained by MRI and PET (positron emission tomography). The combination of different image intrinsic characteristic from different imaging system can improve the ability of accurate lesion region determination and localization.

We have developed a method that allow us to register 3D multi-modalities imaging sets automatically. This method use the N-bar landmarks to solve the transformation parameters from one imaging coordination to a common physical coordination. Using these parameters, the work of $3 \mathrm{D}$ imaging registration can be achieved by coordinations transformations with a simple matrix operations.

In order to analyze the fused 3D imaging sets, we have developed a WINDOWS-95 based software for routine clinical diagnosis. With friendly operation, this software can interactively display the registered imaging at an arbitrary slice in real time.

\section{Methods}

Imaging Registration The imaging registration is achieved with two external $\mathrm{N}$-bar landmarks which is fixed beside patient's head during scanning. The $\mathrm{N}$-bar landmarks are constructed by tubes that is filled with cooper sulfate(MR) or ${ }^{18} \mathrm{~F}$-deoxyl glucose(PET). The transformation parameters from one imaging coordination to a common physical coordination, which are defined to match different imaging system, include scaling, translation and rotation on each axis. The scaling parameters can be obtained from scanning conditions such as FOV, slice thickness. For solving the other parameters, a computer program has been developed. This program includes three procedures: Firstly, it identifies the positions of external fiducial N-shape landmarks on each image slice. Secondly, it fits these landmark locations onto four vertical lines and one oblique plane with linear regression method. The rotation parameters are the three directional angles of the $\mathrm{N}$-bar landmarks. Thirdly, it solves four intersections with vertical lines and oblique plane to find the geometric center of the two N-bar landmarks. We define the translation parameters is same as the coordinate of these N-bar 
geometric center.

Image Presentation For presenting the fused MR and PET imaging, the software transfers the image coordinations into a pre-defined physical coordination(Fig. 2). The transformation parameters such as scaling, rotation and translation are described as $4 \times 4$ matrix $S_{M}, R_{M}, T_{M}$ (for $M R$ imaging) and $S_{p}, R_{p}, T_{p}$ (for PET imaging) since the complete transformation matrix between imaging and physical coordination can be defined as $S_{M} * R_{M} * T_{M}$. Then, a screen coordination is used to view an arbitrary slice from 3D fused imaging sets in physical coordination. The viewing slice is selected by adjusting the scaling $S_{S}$, rotation $R_{S}$ and translation $T_{S}$ matrixes that can be interactively manual driver by a drawing mouse.

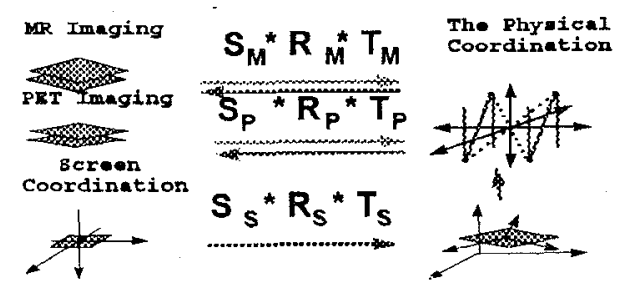

Fig. 2 Imaging Transformation

\section{Results}

Mooncalf A patient is scanned with MR and PET $\left({ }^{18} \mathrm{~F}\right.$ deoxyl glucose)respectively(Fig. 3). In MR imaging, it doesn't show any abnormal in the brain(bottom row). But in PET imaging(top row), an uneven color in the brain indicates

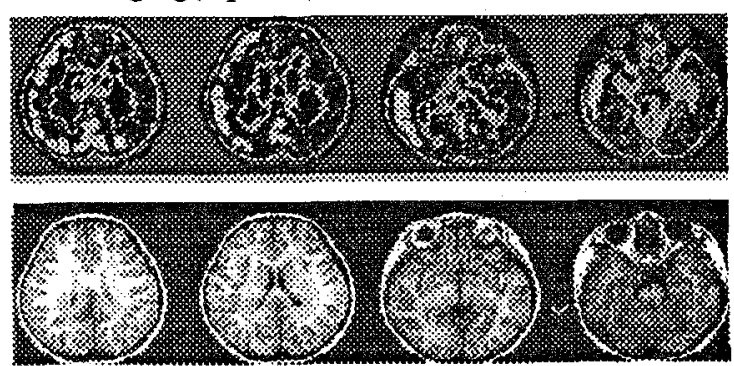

Fig. 3 Fused MR and PET images(top row) and MR images(bottom row) that glucose consumption at left brain is much higher than that of the right brain.

\section{Disscusion}

Qualitative Analysis The MR(Fig. 4,top left), PET(bottom left) and the fused(right) imaging are selected in a common physical coordination to qualitatively show the performance
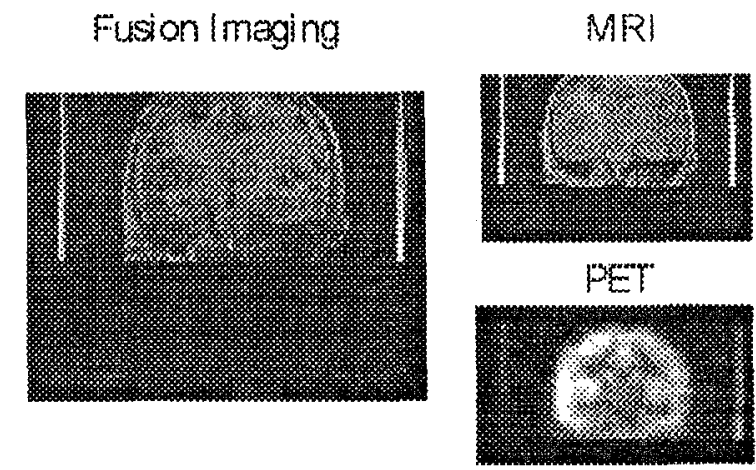

Fig. 4 The Coronal view of N-bar landmarks

of this registration technique. Two lines in MR and PET images represent the pattern of the N-bar landmarks. These pictures verify that the viewing slice of MR and PET imaging are at the same physical plane. It means that their transformation parameters are accurately determined.

\section{Conclusion}

This localization technique allows the fused image set to be interactively displayed friendly at arbitrary plane under WINDOWS-95 environments. The accuracy and reproducibility of slice selection and geometric localization by the technique is within $1.5 \mathrm{~mm}$ and $1^{\circ}$. Clinical rcsults show that this tcchnique is very useful and accurate in diagnosis.

\section{References}

1. Mark W. Wilson, James M. Mountz. A reference system for neuroanatomical localization on functional reconsinucted cerebral images. J Comput Assist Tomogr 1989;13:174-78. 